\title{
1 Higher and deeper: Bringing layer fMRI to association cortex
}

3 Emily S. Finn ${ }^{1,2 *}$, Laurentius Huber ${ }^{3}$, Peter A. Bandettini ${ }^{1}$

$5 \quad{ }^{1}$ Section on Functional Imaging Methods, Laboratory of Brain and Cognition, National Institute

6 of Mental Health, Bethesda, Md., USA

$7 \quad{ }^{2}$ Department of Psychological and Brain Sciences, Dartmouth College, Hanover, NH USA

$8{ }^{3}$ MR-Methods Group, Maastricht Brain Imaging Center, Department of Cognitive Neuroscience,

9 Faculty of Psychology and Neuroscience, Maastricht University, Maastricht, The Netherlands

11 *Corresponding author: emily.finn@nih.gov; emily.s.finn@,dartmouth.edu

14 Abstract: Recent advances in fMRI have enabled non-invasive measurements of brain function in 15 awake, behaving humans at unprecedented spatial resolutions, allowing us to separate activity in 16 distinct cortical layers. While most layer fMRI studies to date have focused on primary cortices, 17 we argue that the next big steps forward in our understanding of cognition will come from 18 expanding this technology into higher-order association cortices, to characterize depth-dependent 19 activity during increasingly sophisticated mental processes. We outline phenomena and theories 20 ripe for investigation with layer fMRI, including perception and imagery, selective attention, and 21 predictive coding. We discuss practical and theoretical challenges to cognitive applications of layer

22 fMRI, including localizing regions of interest in the face of substantial anatomical heterogeneity 23 across individuals, designing appropriate task paradigms within the confines of acquisition 24 parameters, and generating hypotheses for higher-order brain regions where the laminar circuitry 25 is less well understood. We consider how applying layer fMRI in association cortex may help 26 inform computational models of brain function as well as shed light on consciousness and mental 27 illness, and issue a call to arms to our fellow methodologists and neuroscientists to bring layer 28 fMRI to this next frontier. 
A major outstanding challenge in neuroscience is to integrate across levels of investigation, linking

30 genes, molecules, cells, microcircuits, regions, systems and behavior. This will require bringing

31 together evidence from sources across different spatial scales-from the microscopic, such as

32 electrophysiological recordings in animals, to the macroscopic, such as conventional

33 neuroimaging in humans. The mesoscale technique of depth-dependent fMRI, or "layer fMRI"1,

34 which can be applied non-invasively in awake, behaving humans, is a critical missing link to bridge

35 this gap. In this perspective, we argue that layer fMRI, having established that it can reliably detect

36 expected patterns of activity in primary cortex, is now ready to tackle mechanisms of higher-order cognition in association regions.

39 Cortical gray matter is organized into layers with distinct cytoarchitecture, connectivity, and 40 function. In the canonical model of hierarchical connectivity, feedforward connections (i.e., those

41 from lower to higher regions) terminate predominantly in the granular layer (layer IV), while

42 feedback connections (from higher to lower regions) terminate predominantly in infragranular ( $\mathrm{V}$ 43 and VI) and supragranular (I-III) layers (Felleman and Van Essen, 1991; Maunsell and van Essen,

44 1983; Rockland and Pandya, 1979). While this influential model appears to broadly describe the 45 organization of mammalian visual systems (where it was initially characterized), more recent 46 evidence suggests that patterns of laminar connectivity may deviate from this canonical model in 47 other systems and/or further up the cognitive hierarchy-i.e., in regions of association cortex 48 (Barbas, 2015; Godlove et al., 2014; Markov et al., 2014; Rockland, 2019). There may also be 49 subtle but important differences in the organization of these circuits between humans and even our 50 closest evolutionary neighbors, making it yet more imperative to complement animal work with 51 human studies.

\footnotetext{
${ }^{1}$ Note that we use "layer fMRI" throughout this article to mean spatial resolutions capable of resolving depth-dependent activity, but not necessarily individual cortical layers as defined cytoarchitectonically. The current state of the art typically allows for separating signals into two or three compartments, consisting of "superficial" (approximately corresponding to supragranular layers I-III) and "deeper" (infragranular layers V-VI) layers, or "superficial", "middle" (granular layer IV), and "deeper". Advances in acquisition and analysis strategies may allow for future studies will to resolve individual cortical laminae.
} 
52 To date, nearly all layer-fMRI studies have focused on unimodal cortex: visual (Kok et al., 2016;

53 Muckli et al., 2015; Scheeringa et al., 2016), auditory (De Martino et al., 2015; Moerel et al.,

54 2019), motor (Huber et al., 2017; Persichetti et al., 2020), and somatosensory (Yu et al., 2019) -

55 though see Koster et al. (2018), Finn et al. (2019), and Sharoh et al. (2019) for exciting work in

56 the hippocampus, dorsolateral prefrontal cortex, and the occipito-temporal sulcus, respectively.

57 Many use task paradigms that are expected to modulate the balance of feedforward and feedback

58 influences to primary cortex. Feedforward activity is assumed to preferentially target middle

59 layers, while feedback is assumed to preferentially target either superficial or deeper layers or both,

60 depending on the particulars of the paradigm and the areas involved. These studies have been a

61 fruitful proving ground for layer fMRI: when the signals we observe follow the expected patterns,

62 it helps convince us that they are robust and neural in origin. However, ultimately, studying

63 information transfer along the cortical hierarchy by observing only primary cortex is akin to trying

64 to infer the content of a phone conversation by listening to only one side. We argue that layer fMRI

65 is now at a point where it is possible - and indeed, desirable - to move beyond primary cortex into

66 higher-order regions of association cortex. ${ }^{2}$

\section{1. Potential applications of layer fMRI in higher-order brain regions}

68 What types of cognitive phenomena might we investigate with layer fMRI? Below, we outline

69 three domains where applying layer fMRI to association cortex could yield new insights into high-

70 order cognition.

\section{$71 \quad 1.1$ Perception and imagery}

72 Memory and imagery share many of the same brain regions and general mechanisms as perception:

73 for example, in the visual system, simply remembering or imagining something activates areas of

74 visual cortex in a grossly similar way as actually perceiving something via retinal input (Dijkstra

75 et al., 2019; Kosslyn et al., 2001). Yet activity patterns associated with perception and imagery are

\footnotetext{
${ }^{2}$ It is our view that a complete understanding of the role of laminar circuitry in supporting cognition will ultimately require a whole-brain systems perspective, integrating across primary, association, subcortical, and cerebellar regions. In an attempt to keep this article clear and focused, we chose to concentrate on association cortex in particular, since these regions carry some unique challenges and opportunities for experimental design, acquisition and analysis.
} 
not identical (Lee et al., 2012), and longstanding neuropsychological evidence from patients with

77 cortical damage suggests the two are dissociable (Butter et al., 1997; Sirigu and Duhamel, 2001).

78 More recent work suggests that there are subtle differences between laminar patterns of activity in

79 perception versus imagery in primary cortex (van Kerkoerle et al., 2017). At the same time, there

80 is clear evidence that association cortex - particularly prefrontal and parietal areas - has a role in

81 generating and maintaining imagined representations (Nobre et al., 2004), particularly as they

82 relate to task demands (Bugatus et al., 2017; Lee et al., 2013). A complete understanding of

83 perception and imagery, and what distinguishes them, will require measuring simultaneously from

84 primary cortex and higher-order cortex, as many differences could be encoded not only in laminar

85 activity patterns in primary cortex (Persichetti et al., 2020; Turner, 2016), but also — or uniquely—

86 in laminar activity patterns in higher-order regions, and/or layer-specific interactions between

87 primary and higher-order regions. Simultaneous measurements from both primary and higher-

88 order regions would help validate and extend longstanding claims about the roles of bottom-up

89 versus top-down processes in perception and imagery, respectively (Dentico et al., 2014; Dijkstra

90 et al., 2019; Mechelli et al., 2004).

91

92

93

94

95

96

97

98

99

100

101

102

103

\subsection{Attention}

Another phenomenon that could benefit from study with layer-specific tools is attention. Attention is one of the most powerful ways we can modulate our own sensory responses to the external world. Many studies have capitalized on this by manipulating selective attention and observing effects on spatiotemporal patterns of cortical activity to otherwise identical stimuli (De Martino et al., 2015; Gau et al., 2020; Guo et al., 2020; Klein et al., 2018). Yet to date nearly all layer-specific studies of attention record exclusively in primary or unimodal cortex, whereas top-down attentional control is thought to originate from high-order areas in prefrontal and parietal cortex (Squire et al., 2013). Understanding how attention is implemented in the dynamics of top-down and bottom-up pathways, as well as refining and expanding upon computational models of attention (Corchs and Deco, 2002), will require empirical measurements from both higher and lower areas.

\subsection{Predictive coding}


104 Yet a third concept, related in broad strokes to the phenomena discussed above, is predictive 105 coding and other hierarchical theories of brain function (Friston, 2005; Rao and Ballard, 1999).

106 These theories hold that the brain is fundamentally a prediction engine: higher areas generate 107 predictions and relay them to lower areas via feedback connections, while lower areas receive 108 sensory inputs and send them, along with prediction errors, up the hierarchy via feedforward 109 connections. Feedforward and feedback paths are distinguished by cortical depth: feedforward 110 connections originate from superficial layers and terminate in middle layers, while feedback 111 connections predominantly arise from lower layers and terminate in both superficial and deep 112 layers, avoiding middle layers (Bastos et al., 2012). While these theories are compelling and 113 influential, many of their tenets - including the assumed laminar circuitry — still await direct 114 empirical verification (Stephan et al., 2017).

115 Layer fMRI studies have begun to develop paradigms and test hypotheses consistent with the 116 predictive coding framework (Kok et al., 2016; Muckli et al., 2015). But to date, nearly all work 117 has been focused on primary cortex, leaving authors to merely speculate as to the source region(s) 118 giving rise to the predictions, and the intrinsic dynamics of these prediction-generating regions.

119 Now is an opportune time for studies situated within the framework of predictive coding to 120 leverage fMRI's main advantage - its wide-field capacity - to extend their measurements to 121 association regions, and thereby achieve a more complete picture of predictive circuits.

\section{2. Methodological considerations for layer fMRI in higher-order regions}

124 Layer fMRI in association cortex is quite achievable, though not without difficulties. Below, we 125 show preliminary data demonstrating this feasibility, as well as briefly review challenges and 126 potential solutions. These include practical challenges to acquiring and analyzing data, as well as 127 theoretical challenges to generating hypotheses, choosing experimental paradigms, and 128 interpreting results.

130 Note that there are several outstanding issues and limitations to layer fMRI in general, including 131 issues with distortion, smoothing, and achieving spatial specificity in the face of venous artifacts 132 at the cortical surface. These challenges, and methods to overcome them, are reviewed extensively 
133 elsewhere (Kashyap et al., 2018; Polimeni et al., 2018), including other articles in this issue. Here,

134 we focus on additional considerations that arise when applying layer fMRI in association cortex.

\section{Example results of layer-fMRI in association cortex}

selected ICA components in association cortex

7T Magnetom, NIH; two concatenated runs of rest, each 44 min; $0.8 \mathrm{~mm}$ iso voxels; $\mathrm{TR}=2.7+2 \mathrm{~s}$; SS-SI-VASO, 3D-EPI, GRAPPA 3; underlay: $0.5 \mathrm{~mm}$ MP2RAGE.
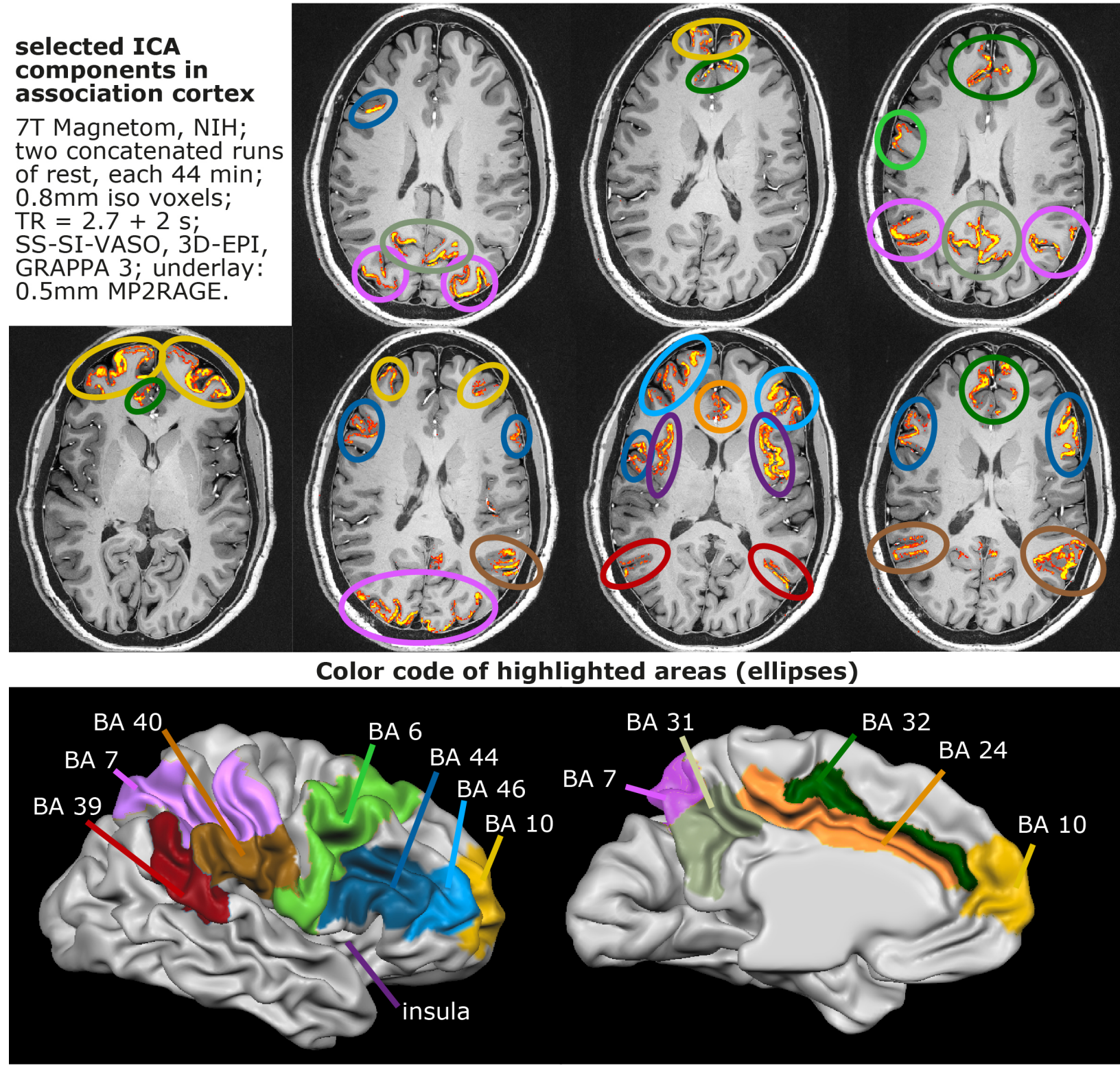

Figure 1. Feasibility of layer fMRI in association regions.

The purpose of this figure is to show that state-of-the-art layer-fMRI sequences are capable of capturing submillimeter (voxel size $0.8 \mathrm{~mm}$ isotropic) brain activity across many higher-order brain areas. Data shown here are from a single representative participant of a larger study described in Huber et al. (this issue). Data were acquired on the 7T Magnetom in the Section on Functional Imaging Methods at the National Institute of Mental Health, with an SS-SI-VASO sequence (slice-saturation, slab-inversion, vascular space occupancy) during an 89min functional resting-state experiment. 


\subsection{Feasibility of layer fMRI in association cortex}

137 We include here some preliminary data showing feasibility of measuring depth-dependent signal

138 in higher-order brain regions. Figure 1 shows data from a recent resting-state experiment acquired

139 at $7 \mathrm{~T}$ using a state-of-the-art functional sequence with $0.8 \mathrm{~mm}$ isotropic voxels. The data-driven

140 approach of independent components analysis (ICA) helps avoid the need for a priori ROIs, which

141 can be more challenging to define in non-primary regions (discussed further in the following two

142 sections). ICA yields signals that appear clean and reasonable (Fig. 1) - i.e., they are localized to

143 gray matter, and are consistent with known functional networks involving higher-order regions

144 (e.g., default mode, frontoparietal, salience). This helps give us confidence that layer-dependent 145 signals can be recovered both outside of primary cortex and in the absence of strong task-evoked

146 modulation. Figure 2 explores these same data in further detail to show that different regions of 147 association cortex have different layer profiles - in other words, overall signal of a region may be 148 dominated by superficial, middle, or deeper layers, or some combination. 




Figure 2. Exploring layer profiles for various regions of association cortex.

This figure demonstrates the variability of layer-fMRI results across the wide spectrum of higher-order brain areas. The left two columns ("functional maps") highlight various layer-specific activity features across brain areas; some areas show largest signal activities in the superficial layers (e.g., Brodmann area [BA] 7), some areas show largest signals in the deeper layers (e.g., BA 40), some areas show both (e.g., BA 10), and some areas have rather unspecific responses (e.g., insula). The corresponding red layer-profiles (middle graphs) refer to the overall layer-dependent fluctuation power of this brain area (note, this does not require the manual selection of ICAs). These data refer to the same study that is mentioned in Fig. 1. The right column ("ex-vivo normalization") depicts how the structural layer location is also highly variable across association areas. The thicknesses and the location of each cytoarchitectonically defined cortical layer are slightly different. Thus, for proper interpretation of the depth-dependent functional results, ex-vivo atlases and depth-normalizations are vital. Here, the thickness and location of each cytoarchitectonical cortical layer is normalized based on the FLASH (TE=60ms) and histology (SMI-32) atlas from Ding et al. (2016).

149 Figure 2 also highlights the need for region-specific normalization of MRI-based "layers" with 
150 ground-truth cytoarchitecture, since, just as in primary cortex, the position and relative thickness 151 of different layers can vary substantially across regions of association cortex. In light of this 152 variation, accurately visualizing and interpreting depth-dependent fMRI signals relies heavily on 153 evidence from histology as well as in vivo and ex vivo anatomical imaging techniques such as 154 diffusion MRI (Roebroeck et al., 2019), T1-weighted imaging for myeloarchitecture (Dinse et al., 155 2015), and magnetic susceptibility imaging (Deistung et al., 2013). Ongoing efforts to create 156 whole-brain laminar atlases with increasing detail and resolution (Trampel et al., 2019; Wagstyl 157 et al., 2020) will greatly benefit depth-dependent functional imaging in association cortex as well 158 as across the whole brain.

\subsection{Practical challenges: Acquisition}

161 Association cortex, especially the parietal and prefrontal lobes, is highly variable across 162 individuals in both structure and functional anatomy (Mueller et al., 2013). Unlike primary regions 163 that often have identifiable landmarks, such as the Stria of Gennari in the primary visual cortex or

164 'hand knob' in the primary motor cortex, functional subdivisions of association cortex are difficult 165 to pinpoint in individual subjects by macroscale anatomical features alone. This can pose 166 challenges for both acquisition and analysis.

167 Because most of the early layer fMRI sequences could not offer whole-brain coverage at the 168 resolutions necessary to resolve depth-dependent activity, experimenters were limited to a partial 169 field of view, which had to be placed over the desired brain area in real time (i.e., while the 170 participant was in the scanner, before the start of the high-resolution experiments). A misplaced 171 slice prescription could mean failure to optimize signal acquisition in the most important region(s), 172 or missing the area of activation entirely. Yet, especially in association cortex, the lack of 173 anatomical landmarks makes it difficult to place slices based on a structural localizer alone. One

174 solution is to conduct an online functional localizer at standard resolution at the beginning of the 175 scan session, using a similar or identical task paradigm as the one used for high-resolution scans. 176 If the task modulation is sufficiently strong - and it is advisable to use strong tasks for layer 177 fMRI - the real-time general linear model capabilities should reveal the "hotspot" that can be used 178 for subsequent slab positioning. 
Acquisition methods for layer fMRI are improving rapidly, and whole-brain (or near whole-brain)

180 coverage is now possible with certain pulse sequences. Whole-brain acquisitions largely obviate

181 the need for online functional localizers (though if there are substantial signal inhomogeneities

182 across the field of view, experimenters might still find them useful to help optimize signal quality

183 in the main region(s) of interest). But these whole-brain sequences often have caveats: the wide-

184 field spatial resolution may come at the cost of temporal resolution (in the case of cerebral blood

185 volume (CBV)-based sequences e.g., vascular space occupancy (VASO), which requires an

186 inversion pulse and acquisitions interleaved with BOLD), or depth specificity (in the case of GE-

187 BOLD, which is prone to draining vein artifacts at the cortical surface). Long TRs may be

188 acceptable for certain paradigms, but others may require a faster sampling rate, especially the

189 event-related designs that are often best suited for probing nuanced cognitive processes (discussed

190 further below). Our current recommendation is that experimenters consider tradeoffs between

191 spatial resolution, spatial specificity, imaging field of view, and temporal resolution in selecting

192 the most appropriate acquisition method for their particular study, noting that these tradeoffs may

193 be different for studies of higher-order versus primary cortex.

\subsection{Practical challenges: Analysis}

195 An ongoing challenge to analyzing layer fMRI data from association cortex is how to resolve

196 depth-dependent signals with respect to cortical laminae defined based on cytoarchitecture. While

197 current layer fMRI sequences offer spatial resolutions as high as $0.5 \mathrm{~mm}$, this is not enough to

198 resolve structural landmarks of all six individual cyto-architectonically defined layers with Nyquist

199 sampling in the functional data. In primary cortex, which has been heavily investigated in the last

200 century, a clear correspondence between relative cortical depths and respective partial voluming 201 of canonical laminae has been established, making it relatively straightforward to interpret 202 functional signal in primary areas with respect to true underlying cortical layers. However, in 203 association regions, the relationship between relative depth and underlying layers is highly variable 204 (Wagstyl et al., 2020) and less well investigated. Thus, the interpretation of layer fMRI results is 205 less clear in association cortices compared to primary cortices. Future layer fMRI studies in 206 association cortex will need to build additional groundwork to relate cortical depths to 207 cytoarchitectonic cortical layers. Analogous to our previous study (Finn et al., 2019), this can be 208 achieved using ex-vivo atlases that have both histology and MRI components (Amunts et al., 2013; 
209 Ding et al., 2016). In any case, the increased complexity of the correspondence between cortical 210 depths and cell-type specific layer communications further underscores the importance of applying 211 layer fMRI in association areas.

212 Another challenge to layer fMRI in association cortex concerns how best to model and interpret 213 task-evoked signal changes. Traditional fMRI activation analyses rely heavily on the general linear 214 model (GLM), in which a model of the task is convolved with an assumed hemodynamic response 215 function and compared to observed brain signal timecourses. However, results of depth-dependent 216 GLMs can be hard to interpret for the following reasons: (1) signal amplitude, quality and stability 217 are heterogeneous across cortical depths; (2) the hemodynamic response function varies across 218 cortical depths (Uludağ and Blinder, 2018); and (3) the baseline blood volume distribution varies 219 across the cortical depth. Most layer fMRI studies in primary cortex use block designs (Gau et al., 220 2020; Huber et al., 2017), which are relatively less dependent on the particulars of deconvolution 221 and thus enjoy higher detection power - an important advantage in the signal-starved world of 222 high-resolution fMRI. But to probe increasingly nuanced aspects of cognition in high-order 223 regions, the flexibility of event-related designs can help disentangle neural activity to distinct 224 subprocesses of a complex cognitive function. Event-related designs can also minimize participant 225 habituation and anticipation, which may be more problematic as one moves up the cortical 226 hierarchy. In our recent study (Finn et al., 2019), we used an event-related design where trials were 227 sufficiently long to permit averaging of raw trial timecourses, without the need to deconvolve the 228 hemodynamic response. Then, we could directly compare magnitude and depth-dependent 229 location of evoked activity during different periods within the trial. While more rapid event-related 230 designs may still be tricky, we expect that ongoing efforts to improve temporal resolution during 231 acquisition, as well as refine hemodynamic models for analysis (Havlicek and Uludağ, 2020), will 232 allow us to use deconvolution-based analyses with more confidence. Other ways to mitigate biases 233 of layer-dependent differences in the hemodynamic response function include conducting the 234 GLM analysis based on impulse response functions, or estimating (i.e., calibrating) the layer235 dependent HRF from an independent dataset (Fracasso et al., 2016; Silva et al., 2007). These 236 approaches, however, can come along with more degrees of freedom or longer scan durations.

237 Finally, the need to preserve as much spatial precision as possible in layer-specific fMRI studies 238 make it difficult to perform the nonlinear registration step (e.g., to a template brain) typical of 
standard-resolution fMRI studies. This is a challenge for all layer-fMRI work, but may be even

240 more problematic for studies of association cortex, since these areas are typically more variable in

241 their anatomy across individuals, as discussed above. In many cases, the need for nonlinear

242 registration can be obviated altogether by simply performing analyses in single-subject space.

243 Numerical signals can then be statistically combined at the final step for group-level inferences. If

244 possible, displaying single-subject data in addition to group-level statistics is to be encouraged, to

245 help convey how robust and replicable the phenomena are across subjects (Finn et al., 2019;

246 Sharoh et al., 2019).

\subsection{Theoretical challenges and paradigm selection}

248 Another set of challenges surrounds generating hypotheses to test with layer fMRI in association 249 cortex. In their excellent article, Lawrence et al. (2019) outline many areas where layer fMRI might 250 be brought to bear on pressing questions in cognitive neuroscience. Yet while they provide 251 compelling examples for how to apply the technique to such phenomena as working memory, 252 selective attention, and multisensory integration, most of the specific hypotheses they offer are 253 framed around expected patterns of activity in primary cortex. For example, they note that effects 254 of selective attention in sensory cortex would be expected to be most pronounced in superficial 255 and deep layers (consistent with the feedback input pattern), but stop short of hypothesizing what 256 a layer-specific signature of attention might look like in the higher-level regions that are 257 presumably generating the attention in the first place.

258 In many respects, this is understandable. As mentioned above, it is an open question whether the 259 canonical model of layer-specific inputs and outputs to a cortical column, originally defined in 260 primary cortex, holds true in association cortex, which makes it hard to develop clear hypotheses 261 for these regions. But we caution researchers against avoiding these areas altogether, lest we fall 262 into a chicken-and-egg trap: the only way to increase our understanding of the circuitry is to 263 measure from these regions, attempt to interpret activity patterns in light of existing knowledge, 264 and use empirical data to iteratively refine our theories, hypotheses, and experimental designs. We 265 argue that layer fMRI is now at a point where we can expand from tightly controlled experiments 266 in sensory cortex with clear hypotheses - which were necessary to show feasibility of the 267 technique - to more exploratory, data-driven investigations of functional dynamics both across the 268 cortical hierarchy as well as within higher-order regions themselves. 
269 Open-ended paradigms like resting state and naturalistic tasks are good candidates for such 270 exploratory studies. Resting-state acquisitions can be used to investigate layer-specific functional 271 connectivity both within high-order and between primary and high-order regions (Huber et al., this 272 issue). Data acquired during naturalistic stimulation - e.g., movie watching - lend itself to both 273 connectivity and activation analyses. Notably, an identical "ground truth" stimulus across subjects 274 permits analyses such as inter-subject correlation (Hasson et al., 2004; Nastase et al., 2019), in 275 which an activity timecourse from one subject's brain is correlated with the timecourse from the 276 same spatial location in a second subject's brain (or with a different spatial location, in the case of 277 inter-subject functional connectivity (ISFC; Simony et al., 2016)). In fMRI studies at standard 278 resolution, rich, engaging stimuli tend to synchronize activity across individuals not only in 279 sensory regions, but also in association regions. Layer fMRI studies with naturalistic paradigms 280 can reveal the extent to which this synchrony is layer-specific, and by extension, layer inter-subject 281 functional connectivity (i.e., cross-subject, cross-region, cross-layer correlations) may reveal 282 directed interactions (see Figure 3 for a schematic). Unlike within-subject functional connectivity, 283 in which much of the noise is correlated across spatial locations, inter-subject functional 284 connectivity helps isolate signal that is neural in origin. Just as in other layer fMRI studies, it would 285 be important to use robust functional localizers and perform all layer segmentation in individual 286 subject space. Layer-average signals could then be extracted and used as input to the cross-subject 287 analyses, which would obviate the need to register different brains in high-resolution space and 288 minimize concerns about partial volume effects or anatomical variability across subjects. Future 289 studies could also explore the utility of functional alignment approaches such as hyperalignment 290 (Haxby et al., 2011) or shared response modeling (Chen et al., 2015) to achieve an accurate 291 separation of layer sources and ensure correspondence across subjects.

292 A single time-locked stimulus also enables averaging of timecourses within subjects across 293 repeated viewings, which is not possible with resting-state data and may also improve signal-to294 noise ratio for depth-dependent analyses in individuals. This may be of particular value in 295 association regions, where magnitude of evoked activity tends to be small relative to ongoing 296 spontaneous fluctuations.

297 On a methodological note, invasive recordings in non-human primates can achieve unrivaled 298 precision, but their measurements are typically limited to one or a small number of regions at a 


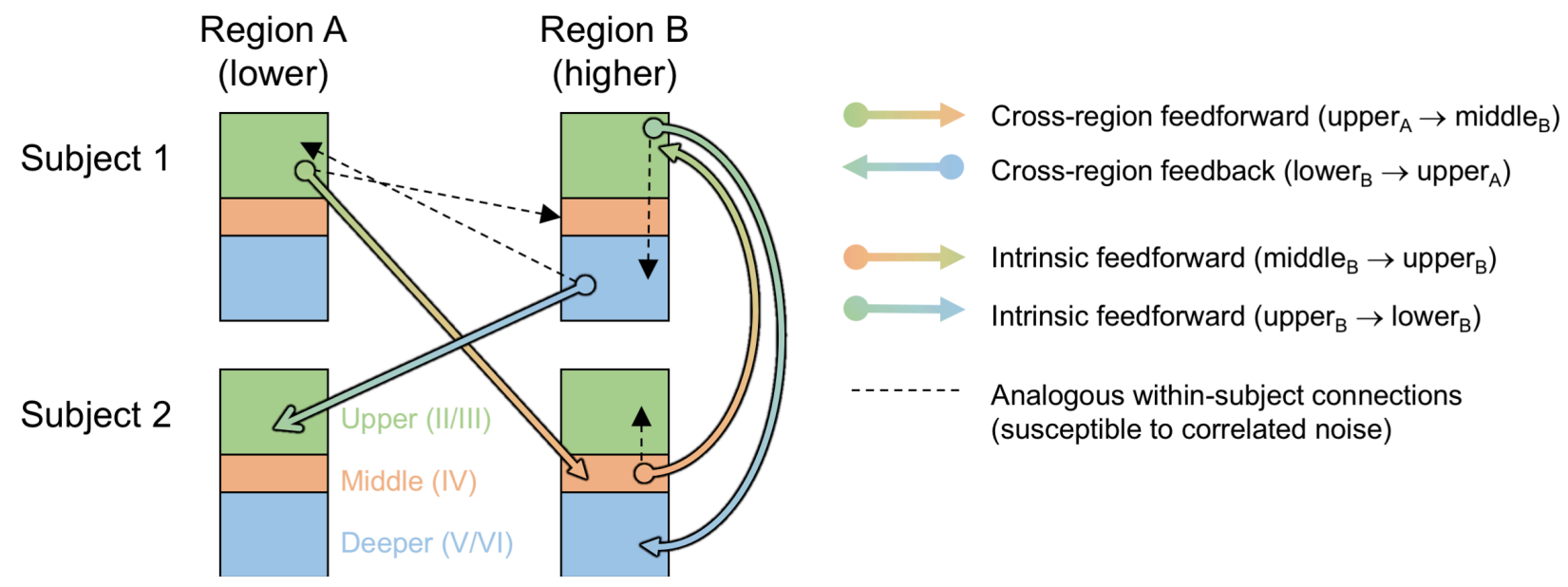

Figure 3. Layer-specific inter-subject functional connectivity. A schematic for how layer-specific ISFC during naturalistic tasks could be used to uncover directed interactions across the cortical hierarchy. Unlike within-subject layer-specific connectivity, cross-subject layer-specific connectivity is expected to be free from correlated noise across layers and regions, meaning it is more likely to be purely neural in origin. Connections of interest are depicted using color-coded arrows, with the hypothesized direction of each connection indicated by the arrowheads. For example, feedforward activity between a lower region (A) and a higher region (B) could be measured by correlating upper layer activity in region A in Subject 1 with middle-layer activity in region B in Subject 2. (Note that the inverse correlations could also be calculated-e.g., from uppera in Subject 2 to middleв in Subject 1 - but are not shown here for clarity). For each connection, the analogous within-subject connection is indicated with a dotted black line. While depicted connections are inspired by the canonical cortical microcircuit model (Bastos et al., 2012), we note again that this model may not fully generalize to laminar circuitry in higherorder regions; therefore, we also suggest data-driven investigations of layer-specific ISFC to identify different or additional connections that may be present within and between these parts of cortex.

time. Layer fMRI is the most promising tool we have for gaining a wide-field view of depthdependent cortical dynamics in many (or, optimistically, all) brain regions simultaneously. We expect that ongoing dialogue between layer fMRI in humans and electrophysiological studies in animal models, where experiments are increasingly using depth-dependent electrodes capable of separating sources from different layers within a cortical column (Bastos et al., 2018; Markowitz et al., 2015), will benefit the field as a whole as we seek to develop and refine models of corticocortical interactions. Specifically, ongoing efforts to benchmark layer fMRI by means of crossspecies comparisons with similar tasks (Self et al., 2019) and concurrent layer-fMRI with electrophysiology (Boorman et al., 2010) will provide more confidence in the neuroscientific interpretability of layer fMRI results. For more in-depth discussion of the validation of layer fMRI and further investigations of linking electrophysiology and layer fMRI, see other articles of this special issue. 
313 In this final section, we consider how bringing layer fMRI to association cortex may help improve

314 computational models of the brain, as well as shed light on some of the biggest questions in

315 neuroscience: what gives rise to conscious experience, and how cortical information processing 316 goes awry in mental illness.

\subsection{Informing macroscale computational brain models}

318 Recent years have seen considerable progress in computational models that simulate macroscale 319 features of brain dynamics using networks of interconnected regions (Deco and Kringelbach, 2014; 320 Gu et al., 2015; Heitmann et al., 2018; Honey et al., 2007; Ritter et al., 2013; Sanz Leon et al., 321 2013). These "synthetic brains" are built by optimizing their fit to empirical data, which typically 322 include a structural backbone measured with anatomical tract imaging (e.g., diffusion tensor 323 imaging), plus functional data from either electromagnetics (e.g., electroencephalography [EEG]), 324 hemodynamics (e.g., fMRI), or both. While these models are increasingly successful at 325 reproducing observed brain dynamics, they are only as good as the empirical data used to develop 326 and refine them. Many models have a strong temporal component, for which EEG is the preferred 327 input modality due to its vastly superior temporal resolution and tighter relationship to neuronal 328 activity. However, one limitation of EEG is that it mainly reflects activity in excitatory superficial329 layer neurons, as these are closest to the skull and oriented perpendicular to the scalp (Buzsáki et 330 al., 2012). Thus, it may give an incomplete picture of cortical dynamics by missing activity in 331 middle and deeper layers; incorporating data from layer fMRI can help fill this gap. A second 332 limitation is that directionality of connections is often inferred from time- or phase-lags between 333 regions, which are much more trustworthy in EEG than in hemodynamic signals (where the slow 334 and spatially variable hemodynamic response function muddies their interpretation), but are 335 nonetheless an indirect measure of causality. Layer fMRI, while also indirect, can provide a 336 complementary source of evidence to help infer directionality based on known laminar-specific 337 patterns of feedforward and feedback activity. In all cases, because intrinsic and cross-regional 338 dynamics can be substantially different between sensory and association regions, it will be critical 339 to incorporate empirical layer fMRI data from both primary and higher-order regions to faithfully 340 synthesize dynamics across the cortical hierarchy. 
342 Influential theories of consciousness posit that awareness arises from the interplay between lower 343 and higher areas; neither is sufficient on its own (Tong, 2003). When consciousness is disrupted-

344 for example, via anesthesia-cortex shows altered activity dynamics both within and across 345 primary and higher-order regions. For example, in animal models, anesthesia substantially alters 346 activity to visual stimulation not only in V1, where it strengthens evoked responses (a quantitative 347 change), but also in PFC, where it dramatically disrupts the pattern of responses (a qualitative 348 change) (Sellers et al., 2013). The functional connectivity (i.e., cross-regional coherence) between 349 the two is also affected, and in both regions, the observed changes are to some extent layer specific 350 (Sellers et al., 2015). In other words, anesthesia does not simply "knock out" prefrontal cortex 351 while leaving unimodal cortex unchanged; rather, it affects both types of regions in terms of both 352 their intrinsic dynamics as well as how they respond to perceptual input. Similarly, lesion studies 353 in humans suggest that both primary and higher-order regions play distinct roles in generating 354 conscious experience (Aru et al., 2012; Koch et al., 2016; Lau and Rosenthal, 2011).

355 If bottom-up sensory signals to primary cortex alone are not enough to evoke consciousness, but 356 rather, awareness stems from top-down recurrent processing of these signals by higher-order 357 regions (Crick and Koch, 2003; Lamme, 2000; Meyer, 2011; Pollen, 1995, 1999), then the 358 resulting signatures of consciousness are expected to show laminar specificity, in accordance with 359 what we know of cortico-cortical circuitry. Layer-fMRI, then, will be an essential tool to advance 360 the study of consciousness (Schneider et al., 2019) — but only if we turn our focus to include 361 association cortex as well as primary regions.

\subsection{Pathology}

363 Many of our most sophisticated cognitive functions - memory, attention, language - are disrupted 364 in mental illness. To the extent that layer fMRI can reveal the circuitry that instantiates these 365 functions, it could also reveal how this circuitry is disrupted in patients suffering from psychiatric 366 conditions (Stephan et al., 2017). These illnesses are complex, and multiple disruptions, either 367 from a common source or distinct sources, may afflict multiple regions along the cortical 368 hierarchy. However, there is longstanding evidence that certain illnesses preferentially affect 
association cortex. Schizophrenia, for example, is associated with pathology in the dorsolateral prefrontal cortex; intriguingly, this pathology often shows a laminar-specific signature. In particular, postmortem studies report that the density and morphology of excitatory pyramidal cells

372 is reduced in layer III (Glantz and Lewis, 2000; Rajkowska et al., 1998), while astroglia are 373 reduced in layer V (Rajkowska et al., 2002). Previous fMRI studies at standard resolution showed 374 altered overall activity levels in dIPFC in patients with schizophrenia (Weinberger et al., 1986), 375 especially during demanding cognitive tasks such as working memory (Cameron S. Carter et al., 376 1998; Perlstein et al., 2001), but were not able to localize disruptions to specific cortical layers. In our recent study, we localized component subprocesses of a working memory task-i.e., delay, response - to different layers within dlPFC in healthy volunteers. An exciting next step would be to conduct this layer-fMRI experiment in patients with schizophrenia, to determine if this layerspecific hypofunctionality can be observed in real time.

Another way in which layer fMRI might advance our understanding of mental illness is by providing clues as to which cortical cell population(s) are dysfunctional. Although isolating activity in specific cell types and/or neurotransmitter systems is typically not possible using traditional endogenous fMRI contrast mechanisms, with layer fMRI we may be able to infer the source of activity at a more mechanistic level than previously possible - e.g., by capitalizing on the fact that cell and receptor types are distributed differently across layers. In combination with work in animal models, this may help to identify and test novel targets for pharmacological intervention. This could also help inform computational brain models discussed above.

Finally, populations with developmental deviations from typical cortical formation, such as those with congenital microcephaly, could provide another valuable window into how layer-specific neural organization supports cognition and behavior. Even mild microcephaly is associated with a

392 reduction in gray matter of up to 40 percent (Sylvester, 1959), and superficial layers may be 393 disproportionately affected (Hammarberg, 1895; Yu et al., 2010). Studying these individuals using 394 layer fMRI techniques could give insight into the degree to which laminar function and circuits 395 are plastic, and, combined with a characterization of cognitive phenotypes, the degree to which 396 their typical organization constrains "normal" brain function. 
399 We believe that layer fMRI, having proven itself robust and reliable in the testing grounds of 400 primary cortex, is now at a point where can and should be brought to bear on some of the biggest 401 questions in cognitive neuroscience. We hope this call to arms inspires other researchers to expand 402 their fields of view, literally and figuratively, into the most highly evolved regions of the brain, to 403 study the underpinnings of our most canonically human capacities.

404 


\section{Acknowledgements}

406 E.S.F. and P.A.B. were supported by the National Institutes of Health (grant ZIAMH002783 to 407 P.A.B.). L.H. was funded from the NWO VENI project 016.Veni.198.032. 
Amunts, K., Lepage, C., Borgeat, L., Mohlberg, H., Dickscheid, T., Rousseau, M.-É., Bludau, S., Bazin, P.-L., Lewis, L.B., Oros-Peusquens, A.-M., et al. (2013). BigBrain: An UltrahighResolution 3D Human Brain Model. Science 340, 1472-1475.

Aru, J., Bachmann, T., Singer, W., and Melloni, L. (2012). Distilling the neural correlates of consciousness. Neurosci Biobehav Rev 36, 737-746.

Barbas, H. (2015). General Cortical and Special Prefrontal Connections: Principles from Structure to Function. Annu Rev Neurosci 38, 269-289. recordings in frontal cortex suggest distinct layers for maintenance and control of working memory. Proc Natl Acad Sci USA, 201710323.

420 Bastos, Andre M., Usrey, W.M., Adams, Rick A., Mangun, George R., Fries, P., and Friston, 421 Karl J. (2012). Canonical Microcircuits for Predictive Coding. Neuron 76, 695-711.

Boorman, L., Kennerley, A.J., Johnston, D., Jones, M., Zheng, Y., Redgrave, P., and Berwick, J. suppression in neurovascular coupling. J Neurosci 30, 4285-4294.

Bugatus, L., Weiner, K.S., and Grill-Spector, K. (2017). Task alters category representations in prefrontal but not high-level visual cortex. Neuroimage 155, 437-449.

427 Butter, C., Kosslyn, S., Mijovic-Prelec, D., and Riffle, A. (1997). Field-specific deficits in visual 428 imagery following hemianopia due to unilateral occipital infarcts. Brain: a journal of neurology 429 120, 217-228.

430 Buzsáki, G., Anastassiou, C.A., and Koch, C. (2012). The origin of extracellular fields and

432 Cameron S. Carter, M.D. ,, William Perlstein, M.D. „, Rohan Ganguli, M.D. ,, Jaspreet Brar, 433 M.D., M.P.H. ,, Mark Mintun, M.D., and, and Jonathan D. Cohen, M.D., Ph.D. (1998).

434 Functional Hypofrontality and Working Memory Dysfunction in Schizophrenia. Am J Psychiatry $435155,1285-1287$.

436 Chen, P.-H.C., Chen, J., Yeshurun, Y., Hasson, U., Haxby, J., and Ramadge, P.J. (2015). A 437 reduced-dimension fMRI shared response model. Paper presented at: Adv Neural Inf Process 438 Syst.

439 Corchs, S., and Deco, G. (2002). Large-scale neural model for visual attention: integration of 440 experimental single-cell and fMRI data. Cereb Cortex 12, 339-348.

441 Crick, F., and Koch, C. (2003). A framework for consciousness. Nat Neurosci 6, 119-126. 
De Martino, F., Moerel, M., Ugurbil, K., Goebel, R., Yacoub, E., and Formisano, E. (2015). Frequency preference and attention effects across cortical depths in the human primary auditory cortex. Proc Natl Acad Sci USA 112, 16036-16041. Computational Connectomics for Understanding Neuropsychiatric Disorders. Neuron 84, 892905.

448 Deistung, A., Schäfer, A., Schweser, F., Biedermann, U., Turner, R., and Reichenbach, J.R. (2013). Toward in vivo histology: A comparison of quantitative susceptibility mapping (QSM) with magnitude-, phase-, and R2 $*$-imaging at ultra-high magnetic field strength. Neuroimage 65 , 299-314.

452 Dentico, D., Cheung, B.L., Chang, J.-Y., Guokas, J., Boly, M., Tononi, G., and Van Veen, B. (2014). Reversal of cortical information flow during visual imagery as compared to visual 454 perception. Neuroimage 100, 237-243.

455 Dijkstra, N., Bosch, S.E., and van Gerven, M.A.J. (2019). Shared Neural Mechanisms of Visual 456 Perception and Imagery. Trends in Cognitive Sciences 23, 423-434.

457 Ding, S.-L., Royall, J.J., Sunkin, S.M., Ng, L., Facer, B.A.C., Lesnar, P., Guillozet-Bongaarts, A., McMurray, B., Szafer, A., Dolbeare, T.A., et al. (2016). Comprehensive cellular-resolution atlas of the adult human brain. J Comp Neurol 524, 3127-3481.

460 Dinse, J., Härtwich, N., Waehnert, M., Tardif, C.L., Schäfer, A., Geyer, S., Preim, B., Turner, R., and Bazin, P.-L. (2015). A cytoarchitecture-driven myelin model reveals area-specific signatures

464 Felleman, D.J., and Van Essen, D. (1991). Distributed hierarchical processing in the primate 465 cerebral cortex. Cerebral cortex (New York, NY: 1991) 1, 1-47.

466 Finn, E.S., Huber, L., Jangraw, D.C., Molfese, P.J., and Bandettini, P.A. (2019). Layer-

467 dependent activity in human prefrontal cortex during working memory. Nat Neurosci 22, 1687 4681695.

469 Fracasso, A., Petridou, N., and Dumoulin, S.O. (2016). Systematic variation of population 470 receptive field properties across cortical depth in human visual cortex. Neuroimage 139, $427-$ 471438.

472 Friston, K. (2005). A theory of cortical responses. Philosophical Transactions of the Royal 473 Society B: Biological Sciences 360, 815-836.

474 Gau, R., Bazin, P.-L., Trampel, R., Turner, R., and Noppeney, U. (2020). Resolving 475 multisensory and attentional influences across cortical depth in sensory cortices. eLife 9, e46856.

476 Glantz, L.A., and Lewis, D.A. (2000). Decreased dendritic spine density on prefrontal cortical 477 pyramidal neurons in schizophrenia. Arch Gen Psychiatry 57, 65-73. 
Godlove, D.C., Maier, A., Woodman, G.F., and Schall, J.D. (2014). Microcircuitry of Agranular Frontal Cortex: Testing the Generality of the Canonical Cortical Microcircuit. J Neurosci 34,

$480 \quad 5355-5369$.

481 Gu, S., Pasqualetti, F., Cieslak, M., Telesford, Q.K., Yu, A.B., Kahn, A.E., Medaglia, J.D., 482 Vettel, J.M., Miller, M.B., Grafton, S.T., et al. (2015). Controllability of structural brain 483 networks. Nature Communications 6, 8414.

484 Guo, F., Liu, C., Qian, C., Zhang, Z., Sun, K., Wang, D.J., He, S., and Zhang, P. (2020). Layer485 dependent multiplicative effects of spatial attention on contrast responses in human early visual 486 cortex. bioRxiv, 2020.2002.2001.926303.

487 Hammarberg, C. (1895). Studien über Klinik und Pathologie der Idiotie, nebst Untersuchungen 488 über die normale Anatomie der Hirnrinde (Berling).

489 Hasson, U., Nir, Y., Levy, I., Fuhrmann, G., and Malach, R. (2004). Intersubject Synchronization

491 Havlicek, M., and Uludağ, K. (2020). A dynamical model of the laminar BOLD response.

492 Neuroimage 204, 116209.

493 Haxby, James V., Guntupalli, J.S., Connolly, Andrew C., Halchenko, Yaroslav O., Conroy, 494 Bryan R., Gobbini, M.I., Hanke, M., and Ramadge, Peter J. (2011). A Common, High495 Dimensional Model of the Representational Space in Human Ventral Temporal Cortex. Neuron $49672,404-416$.

497 Heitmann, S., Aburn, M.J., and Breakspear, M. (2018). The Brain Dynamics Toolbox for 498 Matlab. Neurocomputing 315, 82-88.

499 Honey, C.J., Kötter, R., Breakspear, M., and Sporns, O. (2007). Network structure of cerebral 500 cortex shapes functional connectivity on multiple time scales. Proc Natl Acad Sci USA 104, $501 \quad 10240-10245$.

502 Huber, L., Handwerker, D.A., Jangraw, D.C., Chen, G., Hall, A., Stüber, C., Gonzalez-Castillo, 503 J., Ivanov, D., Marrett, S., Guidi, M., et al. (2017). High-Resolution CBV-fMRI Allows 504 Mapping of Laminar Activity and Connectivity of Cortical Input and Output in Human M1. 505 Neuron 96, 1253-1263.e1257.

506 Kashyap, S., Ivanov, D., Havlicek, M., Poser, B.A., and Uludağ, K. (2018). Impact of acquisition 507 and analysis strategies on cortical depth-dependent fMRI. Neuroimage 168, 332-344.

508 Klein, B.P., Fracasso, A., van Dijk, J.A., Paffen, C.L.E., te Pas, S.F., and Dumoulin, S.O. (2018). 509 Cortical depth dependent population receptive field attraction by spatial attention in human V1. 510 Neuroimage 176, 301-312.

511 Koch, C., Massimini, M., Boly, M., and Tononi, G. (2016). Neural correlates of consciousness: 512 progress and problems. Nat Rev Neurosci 17, 307-321. 
513 Kok, P., Bains, Lauren J., van Mourik, T., Norris, David G., and de Lange, Floris P. (2016).

514 Selective Activation of the Deep Layers of the Human Primary Visual Cortex by Top-Down

515 Feedback. Curr Biol 26, 371-376.

516 Kosslyn, S.M., Ganis, G., and Thompson, W.L. (2001). Neural foundations of imagery. Nat Rev

517 Neurosci 2, 635-642.

518 Koster, R., Chadwick, M.J., Chen, Y., Berron, D., Banino, A., Düzel, E., Hassabis, D., and

519 Kumaran, D. (2018). Big-loop recurrence within the hippocampal system supports integration of

520 information across episodes. Neuron 99, 1342-1354. e1346.

521 Lamme, V.A. (2000). Neural mechanisms of visual awareness: A linking proposition. Brain and

522 Mind 1, 385-406.

523 Lau, H., and Rosenthal, D. (2011). Empirical support for higher-order theories of conscious

524 awareness. Trends in Cognitive Sciences 15, 365-373.

525 Lawrence, S.J.D., Formisano, E., Muckli, L., and de Lange, F.P. (2019). Laminar fMRI:

526 Applications for cognitive neuroscience. Neuroimage 197, 785-791.

527 Lee, S.-H., Kravitz, D.J., and Baker, C.I. (2012). Disentangling visual imagery and perception of 528 real-world objects. Neuroimage 59, 4064-4073.

529 Lee, S.-H., Kravitz, D.J., and Baker, C.I. (2013). Goal-dependent dissociation of visual and 530 prefrontal cortices during working memory. Nat Neurosci 16, 997.

531 Markov, N.T., Vezoli, J., Chameau, P., Falchier, A., Quilodran, R., Huissoud, C., Lamy, C., 532 Misery, P., Giroud, P., Ullman, S., et al. (2014). Anatomy of hierarchy: Feedforward and 533 feedback pathways in macaque visual cortex. J Comp Neurol 522, 225-259.

534 Markowitz, D.A., Curtis, C.E., and Pesaran, B. (2015). Multiple component networks support 535 working memory in prefrontal cortex. Proc Natl Acad Sci USA 112, 11084-11089.

536 Maunsell, J., and van Essen, D. (1983). The connections of the middle temporal visual area (MT) 537 and their relationship to a cortical hierarchy in the macaque monkey. J Neurosci 3, 2563-2586.

538 Mechelli, A., Price, C.J., Friston, K.J., and Ishai, A. (2004). Where Bottom-up Meets Top-down: 539 Neuronal Interactions during Perception and Imagery. Cereb Cortex 14, 1256-1265.

540 Meyer, K. (2011). Primary sensory cortices, top-down projections and conscious experience. 541 Prog Neurobiol 94, 408-417.

542 Moerel, M., De Martino, F., Uğurbil, K., Yacoub, E., and Formisano, E. (2019). Processing 543 complexity increases in superficial layers of human primary auditory cortex. Sci Rep 9, 5502.

544 Muckli, L., De Martino, F., Vizioli, L., Petro, L.S., Smith, F.W., Ugurbil, K., Goebel, R., and 545 Yacoub, E. (2015). Contextual Feedback to Superficial Layers of V1. Current biology : CB 25, $546 \quad 2690-2695$. 
Mueller, S., Wang, D., Fox, Michael D., Yeo, B.T.T., Sepulcre, J., Sabuncu, Mert R., Shafee, R., 548 Lu, J., and Liu, H. (2013). Individual Variability in Functional Connectivity Architecture of the 549 Human Brain. Neuron 77, 586-595.

550 Nastase, S.A., Gazzola, V., Hasson, U., and Keysers, C. (2019). Measuring shared responses 551 across subjects using intersubject correlation. Soc Cogn Affect Neurosci 14, 667-685.

552 Nobre, A.C., Coull, J.T., Maquet, P., Frith, C.D., Vandenberghe, R., and Mesulam, M.M. (2004). 553 Orienting Attention to Locations in Perceptual Versus Mental Representations. J Cogn Neurosci $554 \quad 16,363-373$.

555 Perlstein, W.M., Carter, C.S., Noll, D.C., and Cohen, J.D. (2001). Relation of prefrontal cortex 556 dysfunction to working memory and symptoms in schizophrenia. Am J Psychiatry 158, 11055571113.

558 Persichetti, A.S., Avery, J.A., Huber, L., Merriam, E.P., and Martin, A. (2020). Layer-Specific 559 Contributions to Imagined and Executed Hand Movements in Human Primary Motor Cortex.

560 Curr Biol.

561 Polimeni, J.R., Renvall, V., Zaretskaya, N., and Fischl, B. (2018). Analysis strategies for high562 resolution UHF-fMRI data. Neuroimage 168, 296-320.

563 Pollen, D.A. (1995). Cortical areas in visual awareness.

564 Pollen, D.A. (1999). On the neural correlates of visual perception. Cereb Cortex 9, 4-19.

565 Rajkowska, G., Miguel-Hidalgo, J.J., Makkos, Z., Meltzer, H., Overholser, J., and Stockmeier, 566 C. (2002). Layer-specific reductions in GFAP-reactive astroglia in the dorsolateral prefrontal 567 cortex in schizophrenia. Schizophr Res 57, 127-138.

568 Rajkowska, G., Selemon, L.D., and Goldman-Rakic, P.S. (1998). Neuronal and glial somal size 569 in the prefrontal cortex: a postmortem morphometric study of schizophrenia and Huntington 570 disease. Arch Gen Psychiatry 55, 215-224.

571 Rao, R.P., and Ballard, D.H. (1999). Predictive coding in the visual cortex: a functional 572 interpretation of some extra-classical receptive-field effects. Nat Neurosci 2, 79-87.

573 Ritter, P., Schirner, M., McIntosh, A.R., and Jirsa, V.K. (2013). The virtual brain integrates 574 computational modeling and multimodal neuroimaging. Brain connectivity 3, 121-145.

575 Rockland, K.S. (2019). What do we know about laminar connectivity? Neuroimage 197, 772576784.

577 Rockland, K.S., and Pandya, D.N. (1979). Laminar origins and terminations of cortical 578 connections of the occipital lobe in the rhesus monkey. Brain Res 179, 3-20.

579 Roebroeck, A., Miller, K.L., and Aggarwal, M. (2019). Ex vivo diffusion MRI of the human

580 brain: Technical challenges and recent advances. NMR Biomed 32, e3941. 
Sanz Leon, P., Knock, S., Woodman, M., Domide, L., Mersmann, J., McIntosh, A., and Jirsa, V. 582 (2013). The Virtual Brain: a simulator of primate brain network dynamics. Front Neuroinform 7.

583 Scheeringa, R., Koopmans, P.J., van Mourik, T., Jensen, O., and Norris, D.G. (2016). The 584 relationship between oscillatory EEG activity and the laminar-specific BOLD signal. Proc Natl 585 Acad Sci USA 113, 6761-6766.

586 Schneider, M., Kemper, V.G., Emmerling, T.C., De Martino, F., and Goebel, R. (2019).

587 Columnar clusters in the human motion complex reflect consciously perceived motion axis. Proc 588 Natl Acad Sci USA 116, 5096-5101.

589 Self, M.W., van Kerkoerle, T., Goebel, R., and Roelfsema, P.R. (2019). Benchmarking laminar 590 fMRI: Neuronal spiking and synaptic activity during top-down and bottom-up processing in the 591 different layers of cortex. Neuroimage 197, 806-817.

592 Sellers, K.K., Bennett, D.V., Hutt, A., and Fröhlich, F. (2013). Anesthesia differentially 593 modulates spontaneous network dynamics by cortical area and layer. J Neurophysiol 110, 27395942751.

Sellers, K.K., Bennett, D.V., Hutt, A., Williams, J.H., and Fröhlich, F. (2015). Awake vs. anesthetized: layer-specific sensory processing in visual cortex and functional connectivity between cortical areas. J Neurophysiol 113, 3798-3815.

598 Sharoh, D., van Mourik, T., Bains, L.J., Segaert, K., Weber, K., Hagoort, P., and Norris, D.G. 599 (2019). Laminar specific fMRI reveals directed interactions in distributed networks during 600 language processing. Proc Natl Acad Sci USA 116, 21185-21190.

601 Silva, A.C., Koretsky, A.P., and Duyn, J.H. (2007). Functional MRI impulse response for BOLD 602 and CBV contrast in rat somatosensory cortex. Magnetic Resonance in Medicine: An Official 603 Journal of the International Society for Magnetic Resonance in Medicine 57, 1110-1118.

604 Simony, E., Honey, C.J., Chen, J., Lositsky, O., Yeshurun, Y., Wiesel, A., and Hasson, U. 605 (2016). Dynamic reconfiguration of the default mode network during narrative comprehension. 606 Nature Communications 7, 12141.

607 Sirigu, A., and Duhamel, J. (2001). Motor and visual imagery as two complementary but 608 neurally dissociable mental processes. J Cogn Neurosci 13, 910-919.

609 Squire, R.F., Noudoost, B., Schafer, R.J., and Moore, T. (2013). Prefrontal Contributions to 610 Visual Selective Attention. Annu Rev Neurosci 36, 451-466.

611 Stephan, K.E., Petzschner, F.H., Kasper, L., Bayer, J., Wellstein, K.V., Stefanics, G., 612 Pruessmann, K.P., and Heinzle, J. (2017). Laminar fMRI and computational theories of brain 613 function. Neuroimage.

614 Sylvester, P.E. (1959). Cerebral atrophy in microcephalic cousins. Arch Dis Child 34, 325-330.

615 Tong, F. (2003). Primary visual cortex and visual awareness. Nat Rev Neurosci 4, 219-229. 
616 Trampel, R., Bazin, P.-L., Pine, K., and Weiskopf, N. (2019). In-vivo magnetic resonance 617 imaging (MRI) of laminae in the human cortex. Neuroimage 197, 707-715.

618 Turner, R. (2016). Uses, misuses, new uses and fundamental limitations of magnetic resonance 619 imaging in cognitive science. Philosophical Transactions of the Royal Society B: Biological 620 Sciences $371,20150349$.

621 Uludağ, K., and Blinder, P. (2018). Linking brain vascular physiology to hemodynamic response 622 in ultra-high field MRI. Neuroimage 168, 279-295.

623 van Kerkoerle, T., Self, M.W., and Roelfsema, P.R. (2017). Layer-specificity in the effects of 624 attention and working memory on activity in primary visual cortex. Nature Communications 8 , 62513804.

626 Wagstyl, K., Larocque, S., Cucurull, G., Lepage, C., Cohen, J.P., Bludau, S., Palomero627 Gallagher, N., Lewis, L.B., Funck, T., Spitzer, H., et al. (2020). BigBrain 3D atlas of cortical 628 layers: Cortical and laminar thickness gradients diverge in sensory and motor cortices. PLoS Biol $629 \quad 18, \mathrm{e} 3000678$.

630 Weinberger, D.R., Berman, K.F., and Zec, R.F. (1986). Physiologic Dysfunction of Dorsolateral 631 Prefrontal Cortex in Schizophrenia: I. Regional Cerebral Blood Flow Evidence. Arch Gen 632 Psychiatry 43, 114-124.

633 Yu, T.W., Mochida, G.H., Tischfield, D.J., Sgaier, S.K., Flores-Sarnat, L., Sergi, C.M., Topçu, 634 M., McDonald, M.T., Barry, B.J., Felie, J.M., et al. (2010). Mutations in WDR62, encoding a 635 centrosome-associated protein, cause microcephaly with simplified gyri and abnormal cortical 636 architecture. Nat Genet 42, 1015-1020.

637 Yu, Y., Huber, L., Yang, J., Jangraw, D.C., Handwerker, D.A., Molfese, P.J., Chen, G., Ejima, 638 Y., Wu, J., and Bandettini, P.A. (2019). Layer-specific activation of sensory input and predictive 639 feedback in the human primary somatosensory cortex. Science Advances 5, eaav9053.

640 